\title{
Design of controllers for the damping of torsional oscillations using time-scale decomposition
}

\author{
Y-Y Hsu and L Wang \\ Department of Electrical Engineering. National \\ Taiwan University, Taipei, Taiwan, Republic of \\ China
}

\begin{abstract}
A new technique is presented for the design of an excitation controller and a static VAR compensator which are used to improve the damping characteristics of torsional oscillations in a capacitor-compensated power system. To facilitate the controller design, the system matrix is reduced by means of multi-time-scale decomposition while preserving the mechanical shift modes, which are the ones to be damped by the proposed controller. The parameters of the controller are then determined on the basis of the reduced system by using modal control theory. It is found that model reduction can save the effort in controller design without any significant drift in the eigenvalues of the closed-loop system. To demonstrate the effectiveness of the proposed damping scheme under disturbance conditions, computer-simulated dynamic response tests based on a non-linear system model are also performed. It is concluded that all subsynchronous oscillation modes can be effectively damped by the proposed controller.
\end{abstract}

Keywords: power system stability, torsional oscillation, excitation controller, static VAR compensator, model reduction

\section{Introduction}

Various countermeasures to subsynchronous oscillations in a series capacitor-compensated power system have been proposed ${ }^{1-10}$ since the two shaft failures at Mohave station $^{11}$. The damping scheme to be examined in this paper is a combination of an excitation controller ${ }^{2,3}$ and a static VAR compensator ${ }^{5-10}$

The mathematical model that will be employed for the present study is the IEEE First Benchmark Model ${ }^{12}$, which can be described by a system state equation with 34 state variables. In order to save the effort in controller design, several approaches have been developed to reduce the order of the system. Yan and Yu's reduced model ${ }^{3}$ was obtained by neglecting the turbine and governor equations as well as one small time constant of the exciter and by combining generator and exciter masses together. In addition, the high-pressure turbine mass and the medium-pressure mass were combined. The masses of the

Received: May 1988 two low-pressure turbines (LPA and LPB) were also lumped together. Thus the three oscillation modes $(1,2$ and 5) which are less vulnerable to torsional oscillations were eliminated while the other poorly-damped modes $(0$, 3 and 4) were retained. The main drawback of this approach is the considerable eigenvalue drift during the reduction process. Furthermore, laborious work is needed to compute the equivalent spring coefficient for the lumped mass. Balda et al.$^{4}$ presented an alternative method for model reduction and controller design based on plant input-output behaviour. However, the accuracy of the reduced model depends heavily on the choice of the reduction matrix $\mathbf{R}$ and the weighting matrix $\mathbf{Q}$. Usually, it takes a lot of time before a proper choice for these matrices can be reached.

In this paper a novel approach based on multitime-scale decomposition ${ }^{13,14}$ is employed to reduce the order of the system. Controller design based on the reduced model is then carried out using modal control theory. The accuracy of the reduced model is demonstrated by comparing the eigenvalues of the reduced system and the original system. Time-domain simulations using both linear and non-linear system models are also performed. It is concluded that a very accurate reduced model can be achieved by the proposed model reduction technique. Moreover, the combination of the excitation controller and the static VAR compensator, the parameters of which are determined using the reduced model, can offer satisfactory damping effects for all torsional oscillation modes.

\section{Notation}

Genera
$\mathbf{A}, \mathbf{B}, \mathbf{C}$
$\mathbf{G}(s)$

$\mathbf{H}(s)$
$\omega, \theta$
$\delta$
$R, X$
$B_{\mathbf{L}}, B_{\mathrm{C}}$
$E_{\mathbf{F D}}$

Genera

$\mathbf{A}, \mathbf{B}, \mathbf{C}$

system matrix

transfer function of the system output-input ratio

transfer function of the compensator

rotor speed and rotor angle

generator torque angle

resistance and reactance

susceptances of the inductor and capacitor per unit output voltage of exciter 
$V_{\mathrm{R}} \quad$ voltage regulator output

$V_{x} \quad$ infinite bus voltage

$F \quad$ mechanical torque distribution fraction

$H \quad$ moment of inertia

$K \quad$ spring constant

$K_{\mathrm{P}}, K_{1}, K_{\mathrm{D}} \quad$ PID controller gain

$T_{\omega} \quad$ wash-out term time constant

$T \quad$ torque

$i_{\mathrm{L}} \quad$ transmission line current

$i_{\mathrm{S}} \quad$ current of $B_{\mathrm{L}}$

a actuator signal

$e_{\mathrm{s}} \quad$ voltage of $B_{\mathrm{C}}$

$g \quad$ governor opening

\section{Subscripts}

$\mathrm{d}, \mathrm{q} \quad \mathrm{d}$-axis and q-axis stator quantities

$F \quad$ field circuit quantities

$\mathrm{kd}, \mathrm{fq}, \mathrm{kq}$ damper windings quantities

$\mathrm{H}$ high-pressure turbine quantities

I medium-pressure turbine quantities

A, B low-pressure turbine, A, B quantities

$X \quad$ exciter's mechanical quantities

$\mathrm{S}$ static VAR compensator quantities

E excitation system quantities

L transmission line quantities

Prefix

$\Delta \quad$ linearized quantities

\section{Description of the study system}

The system under study is the IEEE First Benchmark Model $^{2}$, the one-line diagram which is shown in Figure 1. A static VAR compensator ${ }^{15}$ of thyristorcontrolled reactor/fixed capacitor (TCR-FC) type, which is connected to the generator bus via a step-down transformer, is also incorporated into the model. System parameters and operating conditions taken from References 2, 3,12 and 16 are given in Appendix 1.

The eigenvalues of the open-loop system without any controllers are listed in the second column of Table 1 . It can be observed that there exists an unstable SSR mode (mode 3 ) and a mode with poor damping (mode 4) under the given operating condition. As the degree of series compensation $\left(X_{\mathrm{C}} / X_{\mathrm{L}}\right)$ is varied, the real parts of the eigenvalues for the SSR modes will change in the manner shown in Figure 2.

It is concluded from Figure 2 that, of the six modes of concern, only modes 2 and 5 remain stable for any degree of series compensation. Therefore it is necessary to install some kinds of dynamic compensators to stabilize the four unstable modes (modes 0,1,3 and 4). To this end, a combination of an excitation controller and a static VAR compensator, the block diagram of which is shown in Figure 3, will be employed.

Before we tackle the problem of controller design, we first introduce the technique of time-scale decomposition in order to reduce the order of the system so that controller design can be carried out on the basis of a smaller reduced system.

\section{Model reduction using multi-time-scale decomposition}

To reduce the order of the system using time-scale decomposition ${ }^{13,14}$, the state equations of the linearized system are first written as

$$
\begin{aligned}
& \dot{\mathbf{X}}(t)=\mathbf{A X}(t)+\mathbf{B U}(t) \\
& \mathbf{Y}(t)=\mathbf{C X}(t)
\end{aligned}
$$

where

$$
\begin{aligned}
\mathbf{X}= & {\left[\Delta \omega_{\mathrm{H}}, \Delta \theta_{\mathrm{H}}, \Delta \omega_{\mathrm{l}}, \Delta \theta_{\mathrm{l}}, \Delta \omega_{\mathrm{A}}, \Delta \theta_{\mathrm{A}}, \Delta \omega_{\mathrm{B}}, \Delta \theta_{\mathrm{B}},\right.} \\
& \Delta \omega, \Delta \delta, \Delta \omega_{\mathbf{X}}, \Delta \theta_{\mathrm{X}}, \Delta a, \Delta g, \Delta T_{\mathrm{H}}, \Delta T_{\mathrm{l}}, \Delta T_{\mathrm{A}}, \\
& \Delta e_{\mathrm{cd}}, \Delta e_{\mathrm{cq}}, \Delta e_{\mathrm{sd}}, \Delta e_{\mathrm{sq}}, \Delta E_{\mathrm{fd}}, \Delta i_{\mathrm{d}}, \Delta i_{\mathrm{F}}, \Delta i_{\mathrm{kd}}, \\
& \left.\Delta i_{\mathrm{q}}, \Delta i_{\mathrm{fq}}, \Delta i_{\mathrm{kq}}, \Delta i_{\mathrm{Ld}}, \Delta i_{\mathrm{Lq}}, \Delta i_{\mathrm{sd}}, \Delta i_{\mathrm{sq}}, \Delta B_{\mathrm{L}}, \Delta V_{\mathrm{R}}\right]^{\mathrm{T}}
\end{aligned}
$$

is the state vector,

$$
Y=\Delta \omega
$$

is the output signal and

$$
\mathbf{U}=\left[U_{\mathrm{E}}, V_{\mathrm{S}}\right]^{\mathrm{T}}
$$

is the input vector.

It can be observed from Table 1 that the oscillation frequencies of the torsional modes (modes 0-5) to be retained in the final representation are lower than those of some other modes $\left(e_{\mathrm{sd}}, e_{\mathrm{sq}}, \ldots\right)$ but are higher than those of the modes in the other group $\left(a, g, T_{\mathbf{H}}, \ldots\right)$. Thus it is necessary to perform time-scale decomposition twice during the model reduction process. In the first stage the system states are divided into a group of slow states and another group of fast states, with the desired torsional modes included in the group of fast-varying states. The resultant state equations are

$$
\begin{aligned}
& \dot{\mathbf{X}}_{1}(t)=\mathbf{A}_{11} \mathbf{X}_{1}(t)+\mathbf{A}_{22} \mathbf{X}_{2}(t)+\mathbf{B}_{1} \mathbf{U}(t) \\
& \dot{\mathbf{X}}_{2}(t)=\mathbf{A}_{21} \mathbf{X}_{1}(t)+\mathbf{A}_{22} \mathbf{X}_{2}(t)+\mathbf{B}_{2} \mathbf{U}(t) \\
& \mathbf{Y}(t)=\mathbf{C}_{1} \mathbf{X}_{1}(t)+\mathbf{C}_{2} \mathbf{X}_{2}(t)
\end{aligned}
$$

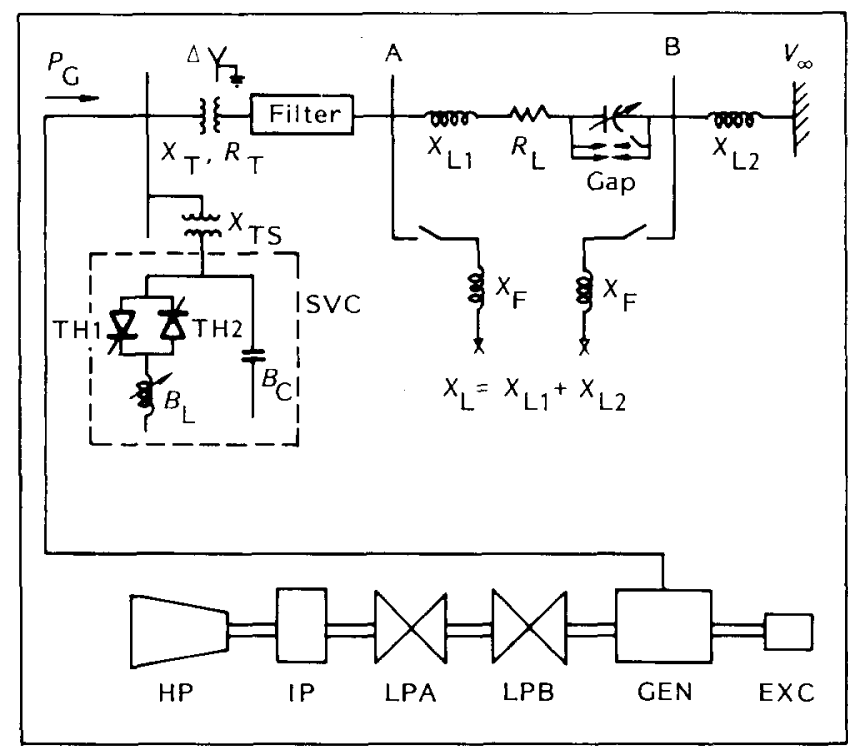

Figure 1. The IEEE First Benchmark Model with a static VAR compensator 
Table 1. System eigenvalues $\left(\mathrm{rad} / \mathrm{s}^{-1}\right)$ under the operating conditions $V_{t}=1.05$ p.u., $P_{G}=0.9$ p.u. and $P F=0.9$ lagging

\section{System conditions}

States

Open-loop system

Open-loop system after time-scale decomposition
System with the PID controller designed using full model
System with the PID controller designed using reduced model

\begin{tabular}{|c|c|c|c|c|c|c|}
\hline \multirow{3}{*}{$\begin{array}{l}\mathbf{X}_{2} \\
\text { states }\end{array}$} & $\begin{array}{l}\mathbf{X}_{3} \\
\text { states }\end{array}$ & $\begin{array}{l}e_{\mathrm{sd}}, e_{\mathrm{sq}} \\
i_{\mathrm{sd}}, i_{\mathrm{sq}} \\
i_{\mathrm{Ld}}, i_{\mathrm{Lq}} \\
i_{\mathrm{d}}, i_{\mathrm{q}} \\
E_{\mathrm{fd}}\end{array}$ & $\begin{array}{l}-3.533 \pm \mathrm{j} 724.704 \\
-0.229 \pm \mathrm{j} 376.99 \\
-3.462 \pm \mathrm{j} 1485.46 \\
-6.964 \pm \mathrm{j} 590.597 \\
-499.933\end{array}$ & $\begin{array}{l}-3.53 \pm \mathrm{j} 724.706 \\
-0.229 \pm \mathrm{j} 376.951 \\
-3.462 \pm \mathrm{j} 1485.46 \\
-6.969 \pm \mathrm{j} 590.597 \\
-499.933\end{array}$ & $\begin{array}{l}-1.301 \pm \mathrm{j} 736.132 \\
-0.242 \pm \mathrm{j} 376.988 \\
-3.983 \pm \mathrm{j} 1479.839 \\
-6.253 \pm \mathrm{j} 590.24 \\
-500.724\end{array}$ & $\begin{array}{l}-1.512 \pm \mathrm{j} 736.974 \\
-0.243 \pm \mathrm{j} 376.987 \\
-3.945 \pm \mathrm{j} 1479.424 \\
-6.197 \pm \mathrm{j} 590.237 \\
-500.720\end{array}$ \\
\hline & $\begin{array}{l}\mathbf{X}_{4} \\
\text { states }\end{array}$ & $\begin{array}{l}\text { Mode } 5: \lambda_{\mathrm{H}} \\
\text { Mode } 4: \lambda_{\mathrm{I}} \\
\text { Mode } 3: \lambda_{\mathrm{A}} \\
\text { Mode } 2: \lambda_{\mathrm{X}} \\
\text { Mode } 1: \lambda_{\mathrm{B}} \\
\text { Mode } 0: \lambda_{\mathrm{G}} \\
V_{\mathrm{R}} \\
e_{\mathrm{cd}}, e_{\mathrm{cq}} \\
i_{\mathrm{F}} \\
i_{\mathrm{kd}} \\
k_{\mathrm{kq}} \\
B_{\mathrm{L}}\end{array}$ & $\begin{array}{l}-0.182 \pm \mathrm{j} 298.178 \\
-0.026 \pm \mathrm{j} 202.695 \\
0.765 \pm \mathrm{j} 160.691 \\
-0.642 \pm \mathrm{j} 172.065 \\
-0.11 \pm \mathrm{j} 99.43 \\
-0.155 \pm \mathrm{j} 9.721 \\
-101.578 \\
-6.984 \pm \mathrm{j} 161.833 \\
-10.293 \\
-31.595 \\
-24.783 \\
-6.0\end{array}$ & $\begin{array}{l}-0.182 \pm \mathrm{j} 298.177 \\
-0.026 \pm \mathrm{j} 202.694 \\
0.765 \pm \mathrm{j} 160.696 \\
-0.642 \pm \mathrm{j} 127.065 \\
-0.11 \pm \mathrm{j} 99.43 \\
-0.156 \pm \mathrm{j} 9.721 \\
-101.578 \\
-6.982 \pm \mathrm{j} 376.951 \\
-10.292 \\
-31.595 \\
-24.783 \\
-5.979\end{array}$ & $\begin{array}{l}-0.182 \pm \mathrm{j} 298.176 \\
-0 . \pm \mathrm{j} 203.2^{*} \\
-2.0 \pm \mathrm{j} 161.9^{*} \\
-0.825 \pm \mathrm{j} 127.064 \\
-2.0 \pm \mathrm{j} 99.9^{*} \\
-3.0 \pm \mathrm{j} 10.0^{*} \\
-121.288 \\
-4.217 \pm \mathrm{j} 161.241 \\
-6.22-\mathrm{j} 0.192 \\
-44.686 \\
-24.788 \\
-6.22+\mathrm{j} 0.192\end{array}$ & $\begin{array}{l}-0.182 \pm \mathrm{j} 298.176 \\
-0.59 \pm \mathrm{j} 203.117 \\
-2.054 \pm \mathrm{j} 160.998 \\
-0.816 \pm \mathrm{j} 127.049 \\
-1.986 \pm \mathrm{j} 99.727 \\
-3.203 \pm \mathrm{j} 9.69 \\
-121.818 \\
-4.246 \pm \mathrm{j} 162.161 \\
-5.721-\mathrm{j} 0.352 \\
-54.893+\mathrm{j} 7.082 \\
-24.787 \\
-5.721+\mathrm{j} 0.352\end{array}$ \\
\hline & \multicolumn{2}{|c|}{ PID controller } & & & $\begin{array}{l}-100.0 \\
-64.209\end{array}$ & $\begin{array}{l}-100.0 \\
-54.893-j 7.082\end{array}$ \\
\hline $\begin{array}{l}\mathbf{X}_{1} \\
\text { states }\end{array}$ & $\begin{array}{l}i_{\mathrm{fq}} \\
T_{\mathrm{H}}, T_{\mathrm{l}}, \\
a, g\end{array}$ & & $\begin{array}{l}-1.705 \\
-0.142 \\
-3.016,-4.477 \\
-4.684 \pm j 0.46\end{array}$ & $\begin{array}{l}-1.705 \\
-0.142 \\
-3.016,-4.459 \\
-4.704 \pm j 0.449\end{array}$ & $\begin{array}{l}-2.063 \\
-0.142 \\
-2.916,-4.743 \\
-4.452 \pm j 1.408\end{array}$ & $\begin{array}{l}-4.782 \\
-0.142 \\
-2.979 \pm j 0.331 \\
-3.639 \pm j 2.422\end{array}$ \\
\hline
\end{tabular}

* Denotes exactly-assigned eigenvalues

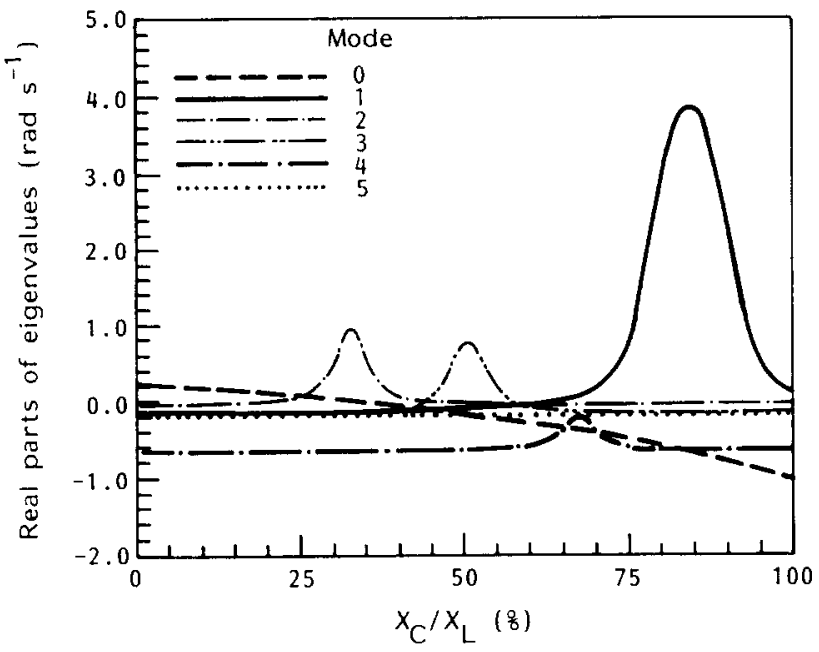

Figure 2. The real parts of the eigenvalues associated with torsional modes for the open-loop system as a function of $X_{C} / X_{L}$

Here $\mathbf{X}_{1}=\left[\Delta a, \Delta g, \Delta T_{\mathrm{H}}, \Delta T_{\mathrm{l}}, \Delta T_{\mathrm{A}}, \Delta i_{\mathrm{fq}}\right]^{\mathrm{T}}$ is the vector comprising all slowly-varying state variables to be reduced and $\mathbf{X}_{2}$ is the vector composed of the other state variables. Using the time-scale decomposition technique described in References 13 and 14, the original system can be decoupled into two subsystems, the fast one being the desired reduced model:

- slow subsystem

$$
\dot{\mathbf{X}}_{1}(t)=\overline{\mathbf{A}}_{11} \mathbf{X}_{1}(t)+\overline{\mathbf{B}}_{1} \mathbf{U}(t)
$$

- fast subsystem

$$
\begin{aligned}
& \dot{\mathbf{X}}_{2}(t)=\overline{\mathbf{A}}_{22} \mathbf{X}_{2}(t)+\overline{\mathbf{B}}_{2} \mathbf{U}(t) \\
& \mathbf{Y}(t)=\overline{\mathbf{C}}_{2} \mathbf{X}_{2}(t)
\end{aligned}
$$

The matrices $\overline{\mathbf{A}}_{11}, \overline{\mathbf{B}}_{1}, \overline{\mathbf{A}}_{22}, \overline{\mathbf{B}}_{2}$ and $\overline{\mathbf{C}}_{2}$ can be computed iteratively from the equations given in Appendix 2 .

To further reduce the order of the model, the system described by equations (7) and (8) is again decomposed into two subsystems, with one fast system $\left(\mathbf{X}_{3}\right)$ comprising the fast-varying state variables to be eliminated and another slow system $\left(\mathbf{X}_{4}\right)$ containing the torsional modes to be retained. The resultant equations are as follows:

- fast subsystem

$$
\dot{\mathbf{X}}_{3}(t)=\overline{\mathbf{A}}_{33} \mathbf{X}_{3}(t)+\overline{\mathbf{B}}_{3} \mathbf{U}(t)
$$


- slow subsystem

$$
\begin{aligned}
& \dot{\mathbf{X}}_{4}(t)=\overline{\mathbf{A}}_{44} \mathbf{X}_{4}(t)+\overline{\mathbf{B}}_{4} \mathbf{U}(t) \\
& \mathbf{Y}(t)=\overline{\mathbf{C}}_{4} \mathbf{X}_{4}(t)
\end{aligned}
$$

Here $\mathbf{X}_{3}=\left[\Delta e_{\mathrm{sd}}, \Delta e_{\mathrm{sq}}, \Delta E_{\mathrm{fd}}, \Delta i_{\mathrm{d}}, \Delta i_{\mathrm{q}}, \Delta i_{\mathrm{Ld}}, \Delta i_{\mathrm{Lq}}, \Delta i_{\mathrm{sd}}\right.$, $\left.\Delta i_{\mathrm{sq}}\right]^{\mathrm{T}}$ is the vector comprising the state variables to be eliminated and $\mathbf{X}_{4}$ is the vector consisting of all the states that are to be retained. Once again, the matrices $\overline{\mathbf{A}}_{33}$, $\overline{\mathbf{B}}_{3}, \overline{\mathbf{A}}_{44}, \overline{\mathbf{B}}_{4}$, and $\overline{\mathbf{C}}_{4}$ can be obtained by the method described in Appendix 2.

Thus the reduced model (equations (10) and (11)) can be reached by two successive time-scale decompositions on the original system (equations (1) and (2)). It should be noted that the fast-slow ratios cannot be fixed in this study since the eigenvalues are spread over a wide range of frequencies. The dynamic response of the decomposed system will differ very much from that of the original system if a fixed fast-slow ratio is employed.

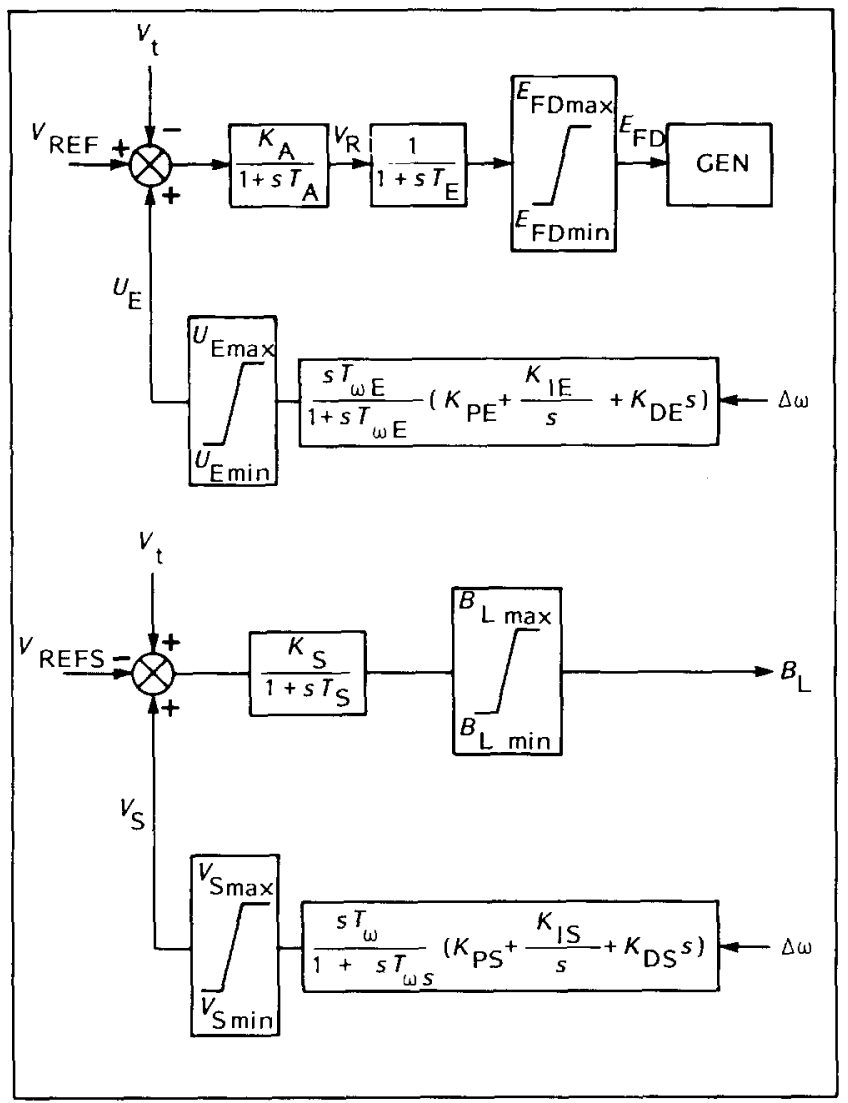

Figure 3. Block diagram of the system with an excitation controller and a static VAR compensator
Before we proceed with the design of controllers using this reduced model, we first have a look at the effect of model reduction on the torsional mode eigenvalues. A comparison of the eigenvalues before and after time-scale decomposition, which are listed in the second and third columns of Table 1 respectively, indicates that the time-scale decomposition does not cause any significant eigenvalue drift.

To give an idea of how the system eigenvalues vary with the iteration process of time-scale decomposition, the eigenvalues for the torsional modes in each iteration during the process of reducing $\mathbf{X}_{3}$ are listed in Table 2. It is observed that the torsional modes which are of primary concern to us have been retained during the reduction process. In addition, the decomposition process can be completed in five iterations.

\section{Controller design using the reduced model}

To determine the parameters of the excitation controller and the static VAR compensator using modal control theory ${ }^{17,18}$, the state equations in the frequency domain are first obtained by taking the Laplace transformation of equations (10) and (11):

$$
\begin{aligned}
& s \mathbf{X}_{\mathbf{4}}(s)=\overline{\mathbf{A}}_{\mathbf{4} 4} \mathbf{X}_{\mathbf{4}}(s)+\overline{\mathbf{B}}_{\mathbf{4}} \mathbf{U}(s) \\
& \mathbf{Y}(s)=\overline{\mathbf{C}}_{\mathbf{4}} \mathbf{X}_{\mathbf{4}}(s)
\end{aligned}
$$

Rearranging equation (12) gives

$$
\mathbf{X}_{\mathbf{4}}(s)=\left(s \mathbf{I}-\overline{\mathbf{A}}_{\mathbf{4 4}}\right)^{-1} \overline{\mathbf{B}}_{4} \mathbf{U}(s)
$$

Substituting equation (14) into equation (13) results in

$$
\mathbf{Y}(s)=\mathbf{G}(s) \mathbf{U}(s)
$$

where

$$
\mathbf{G}(s)=\overline{\mathbf{C}}_{\mathbf{4}}\left(s \mathbf{I}-\overline{\mathbf{A}}_{44}\right)^{-1} \overline{\mathbf{B}}_{\mathbf{4}}
$$

Since the control vector $\mathbf{U}(s)$ can be written as

$$
\mathbf{U}(s)=\left[\begin{array}{c}
U_{\mathrm{E}}(s) \\
V_{\mathrm{S}}(s)
\end{array}\right]=\left[\begin{array}{c}
H_{\mathrm{E}}(s) \\
H_{\mathrm{S}}(s)
\end{array}\right] Y(s)
$$

Mason's rule states that the characteristic equation for the closed-loop system with control signals from the excitation controller (i.e. $U_{\mathrm{E}}(s)$ ) and the static VAR compensator (i.e. $V_{\mathrm{s}}(s)$ ) can be expressed as

$$
\Delta(s)=1-\mathbf{G}(s) \mathbf{H}(s)=0
$$

Table 2. Shaft mode eigenvalues in the iteration process of time-scale decomposition

\begin{tabular}{lllllr}
\hline Iteration & Mode 0 & Mode 1 & Mode 2 & Mode 3 & \multicolumn{1}{l}{ Mode 4 } \\
\hline$k=j=1$ & $-0.159 \pm \mathrm{j} 9.713$ & $-0.119 \pm \mathrm{j} 99.458$ & $-0.637 \pm \mathrm{j} 127.072$ & $0.423 \pm \mathrm{j} 160.448$ & $0.024 \pm \mathrm{j} 202.703$ \\
$k=j=2$ & $-0.162 \pm \mathrm{j} 9.739$ & $-0.106 \pm \mathrm{j} 99.428$ & $-0.641 \pm \mathrm{j} 127.064$ & $0.629 \pm \mathrm{j} 160.847$ & $-0.023 \pm \mathrm{j} 202.678$ \\
$k=j=3$ & $-0.153 \pm \mathrm{j} 9.716$ & $-0.110 \pm \mathrm{j} 99.430$, & $-0.642 \pm \mathrm{j} 127.065$ & $0.756 \pm \mathrm{j} 160.718$ & $-0.024 \pm \mathrm{j} 202.692$ \\
$k=j=4$ & $-0.156 \pm \mathrm{j} 9.724$ & $-0.110 \pm \mathrm{j} 99.430$ & $-0.642 \pm \mathrm{j} 127.065$ & $0.758 \pm \mathrm{j} 160.709$ & $-0.025 \pm \mathrm{j} 292.693$ \\
$k=j=5$ & $-0.156 \pm \mathrm{j} 9.721$ & $-0.110 \pm \mathrm{j} 99.430$ & $-0.642 \pm \mathrm{j} 127.065$ & $0.765 \pm \mathrm{j} 160.696$ & $-0.026 \pm \mathrm{j} 202.694$ \\
\hline
\end{tabular}




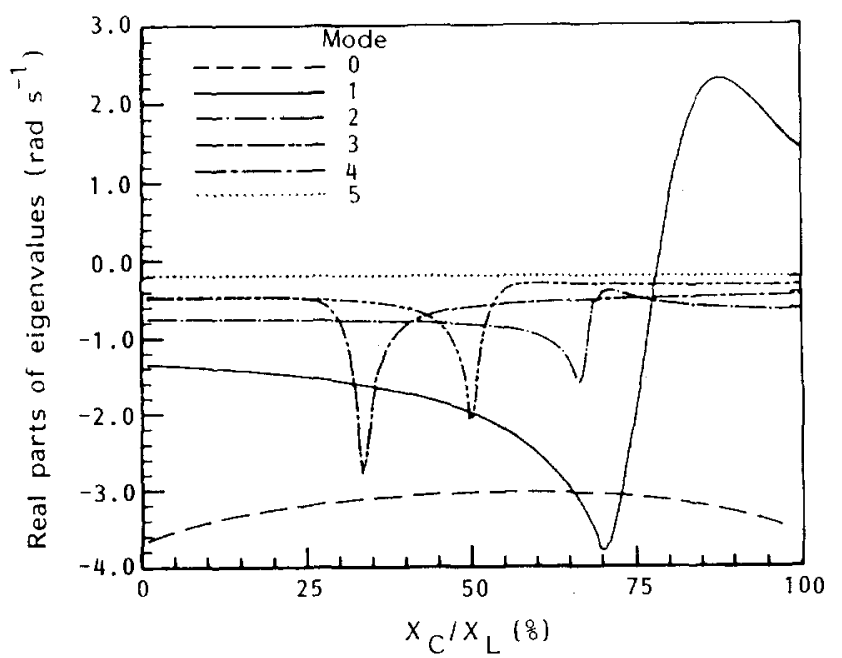

Figure 4. The real parts of the torsional mode eigenvalues as a function of $X_{C} / X_{L}$

where

$$
\mathbf{H}(s)=\left[\begin{array}{l}
H_{\mathrm{E}}(s) \\
H_{\mathrm{S}}(s)
\end{array}\right]
$$

It is observed from Figure 3 that the transfer functions of the excitation controller, $H_{\mathrm{E}}(s)$, and the static VAR compensator, $H_{\mathrm{S}}(s)$, are given by

$$
H_{\mathrm{E}}(s)=\frac{s T_{\omega \mathrm{E}}}{1+s T_{\omega \mathrm{E}}}\left(K_{\mathrm{PE}}+\frac{K_{\mathrm{IE}}}{s}+K_{\mathrm{DE}} s\right)
$$

and

$$
H_{\mathrm{S}}(s)=\frac{s T_{\omega \mathrm{S}}}{1+s T_{\omega \mathrm{S}}}\left(K_{\mathrm{PS}}+\frac{K_{\mathrm{IS}}}{s}+K_{\mathrm{DS}} s\right)
$$

respectively. Note that controllers of the proportionalintegral-derivative (PID) type have been employed in the present study.

When a set of eight desired locations on the complex plane are specified for the eigenvalues associated with the four unstable torsional modes (modes 0, 1, 3 and 4) and are substituted into equation (18), we get a set of eight simultaneous algebraic equations with eight unknown constants $T_{\omega \mathrm{E}}, K_{\mathrm{PE}}, K_{\mathrm{IE}}, K_{\mathrm{DE}}, T_{\omega \mathrm{S}}, K_{\mathrm{PS}}, K_{\mathrm{IS}}$ and $K_{\mathrm{DS}}$. The desired controller parameters can thus be computed by solving these non-linear equations. The results are as follows:

\section{- prespecified eigenvalues}

$$
\begin{array}{ll}
-3.0 \pm \mathrm{j} 10.0 & (\text { mode } 0) \\
-2.0+\mathrm{j} 99.9 & (\text { mode } 1) \\
-2.0 \pm \mathrm{j} 161.9 & (\text { mode } 3) \\
-0.5 \pm \mathrm{j} 203.2 & (\text { mode } 4)
\end{array}
$$

- controller parameters determined by using the reduced model excitation controller

$$
T_{\omega \mathrm{E}}=0.01 \mathrm{~s} \quad \mathrm{~K}_{\mathrm{PE}}=0.68, \quad K_{\mathrm{IE}}=12.24, \quad K_{\mathrm{DE}}=0.06
$$

static VAR compensator

$$
T_{\mathrm{sS}}=0.01 \mathrm{~s}, K_{\mathrm{PS}}=-6.54, K_{\mathrm{IS}}=-18.5, K_{\mathrm{DS}}=-0.1
$$

The eigenvalues of the closed-loop system with the designed controllers installed at the generator are listed in the fifth column of Table 1. It is noted that only insignificant drift in the torsional mode eigenvalues has been observed owing to the model reduction introduced in the controller design.

If controller design is based on the original full system (equations (1) and (2)), the following controller parameters will be reached by using the same prespecified eigenvalues as those employed for the reduced model:

- controller parameters determined by using the full model excitation controller

$$
T_{\omega \mathrm{E}}=0.01 \mathrm{~s}, \quad K_{\mathrm{PE}}=1.43, \quad K_{\mathrm{IE}}=19.82, \quad K_{\mathrm{DE}}=0.06
$$

static VAR compensator

$$
T_{6 \mathrm{~S}}=0.01 \mathrm{~s}, K_{\mathrm{PS}}=-7.87, K_{\mathrm{IS}}=-122.4, K_{\mathrm{DS}}=-0.093
$$

When the controllers with these parameters are installed at the system, the eigenvalues listed in the fourth column of Table 1 will be obtained. The prespecified eigenvalues have been exactly assigned by the designed controllers.

Although controller design using the reduced model will introduce some minor drift in the system eigenvalues, the shift is insignificant. Moreover, the required CPU time for controller design can be reduced from $12.92 \mathrm{~s}$ on a VAX-11/780 computer to $4.76 \mathrm{~s}$ on the same machine if the reduced model is employed to replace the original full model. This is particularly desirable if controller design has to be done many times for different prespecified eigenvalues.

The real parts of the torsional mode eigenvalues for the system equipped with the excitation controller and the static VAR compensator as a function of the degree of series compensation are shown in Figure 4. It is concluded that the closed-loop system, though equipped with a controller designed on the basis of an operating condition of $50 \%$ series compensation, can remain stable as long as $X_{\mathrm{C}} / X_{\mathrm{L}} \leqslant 75 \%$.

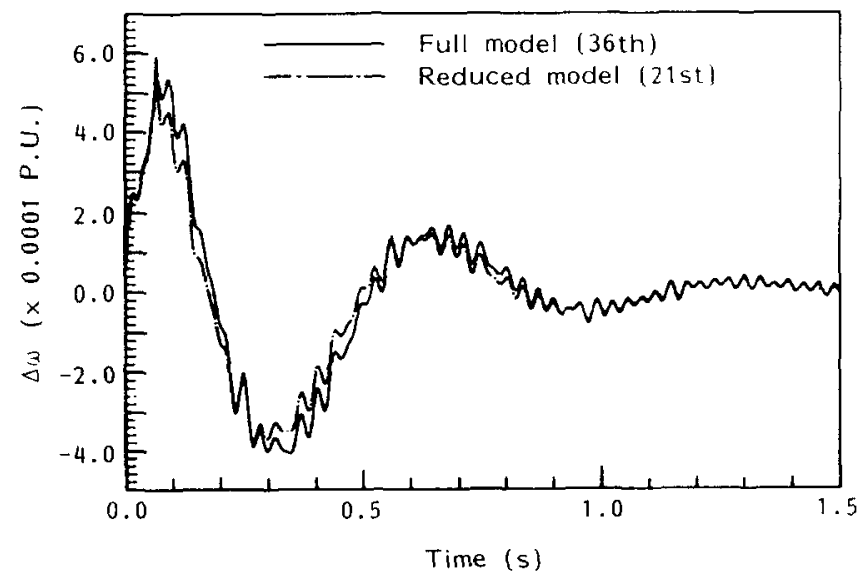

Figure 5. Time-domain simulation results using the linear model 


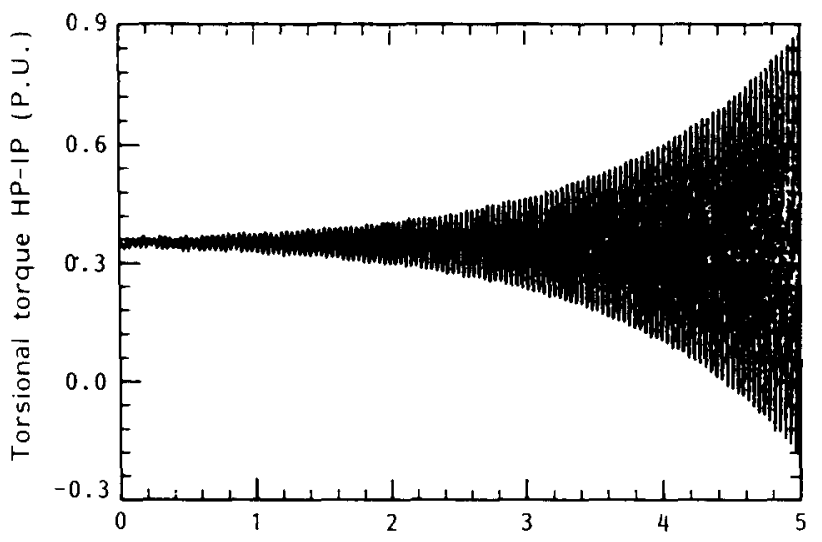

a

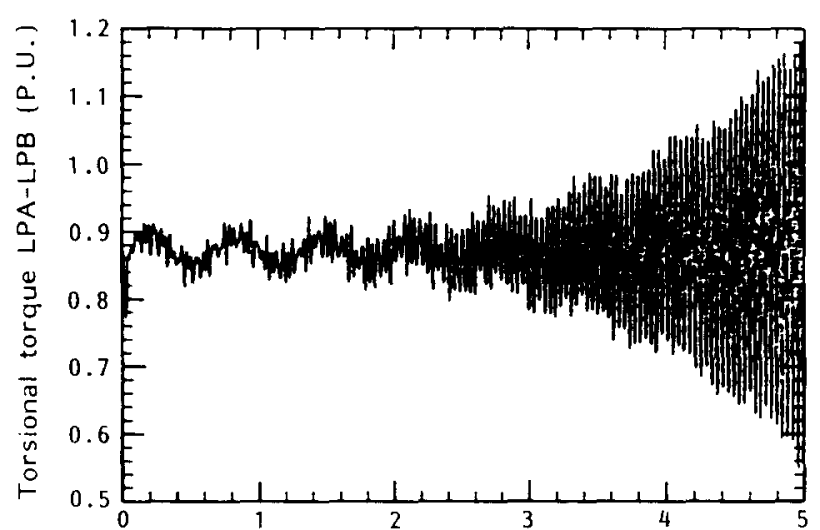

b

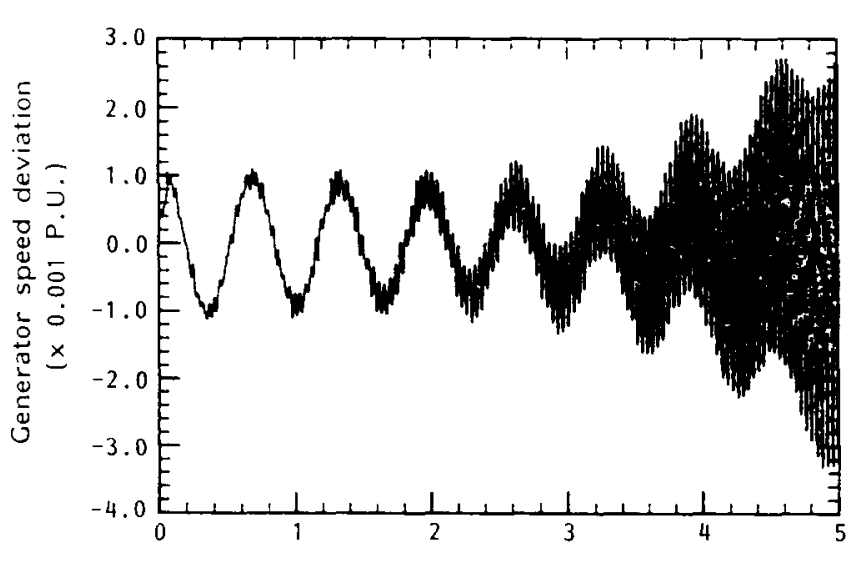

C

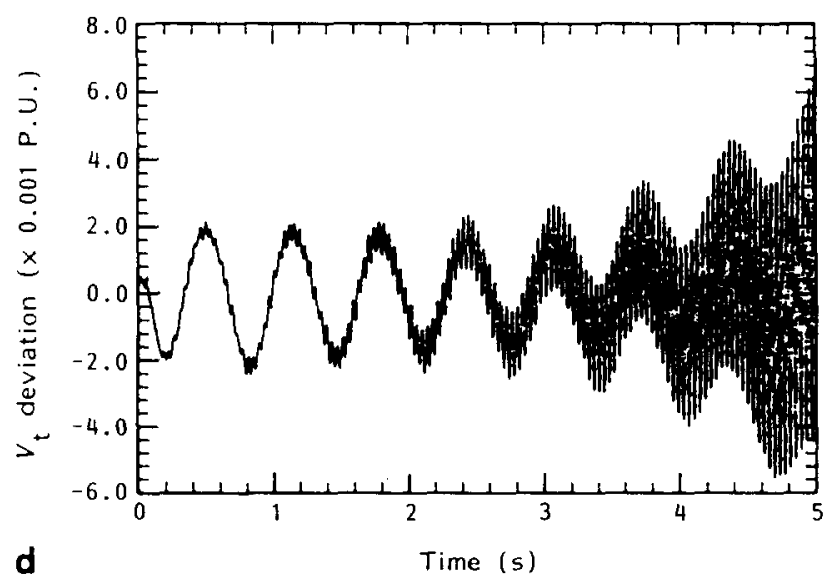

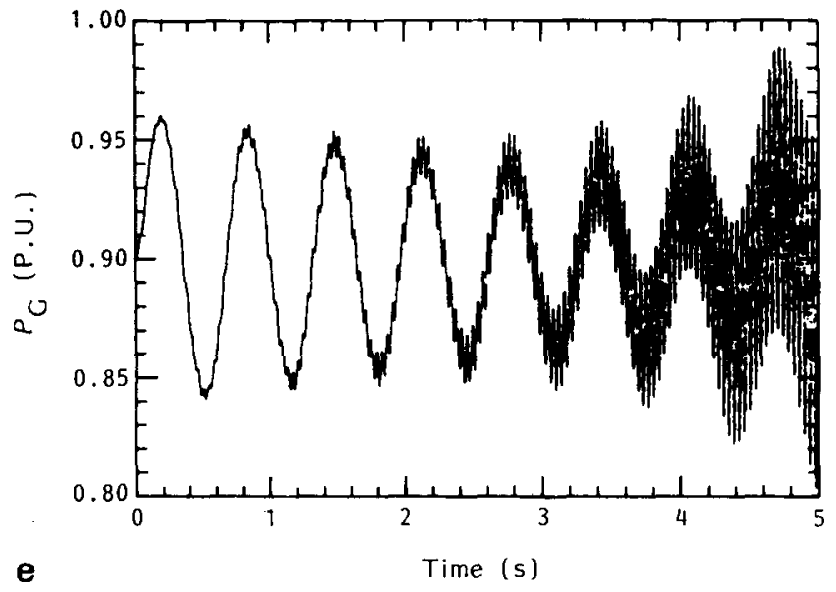

Figure 6. Time-domain simulation results using the non-linear model (open-loop system)

\section{Linear simulation}

It was demonstrated in the last section that as far as system eigenvalues are concerned, the controller designed on the basis of the reduced model will yield almost the same result as that designed using the original system. To look further into the effect of model reduction on system performance, time-domain simulations using both the 36 th-order closed-loop linear system and the 21 st-order reduced closed-loop system were carried out for a pulsed torque disturbance $\left(\Delta T_{\mathrm{e}}\right)$ of $5 \%$ lasting for four cycles. The results are compared in Figure 5. It can be observed from the response curves in Figure 5 that the curve of the reduced model matches very closely that of the full model.

\section{Non-linear simulation}

Another point of interest is the dynamic performance of the system subject to a major disturbance. In this case the system behaviour will be affected by all kinds of non-linearities such as exciter ceilings, control signal limiters, etc. To examine the performance of the controller, which was designed on the basis of a particular operating condition, under these drastically-changing operating conditions, dynamic responses to a $10 \%$, four-cycle pulsed torque $\left(\Delta T_{\mathrm{e}}\right)$ are plotted in Figures 6 and 7. A set of 36th-order non-linear differential equations describing the system behaviour were solved by the fourth-order Runge-Kutta method on a VAX-11/780 computer. Figure 6 depicts the response curves for the open-loop system. Unstable responses have been observed. This is as expected since there exists an unstable torsional mode, as is clear from the eigenvalues listed in the second column of Table 1 . The response curves in Figure 7 indicate that all torsional modes have been effectively damped by the added controller. It can also be concluded from Figure 8 that only small control signals are required by the proposed controller.

\section{Conclusions}

A multi-time-scale decomposition technique has been employed to reduce the order of the system for subsynchronous resonance studies. By separating the fast and slow states, we obtain a reduced model which can yield essentially the same dynamic responses as those obtained by using the original full system. Controller 

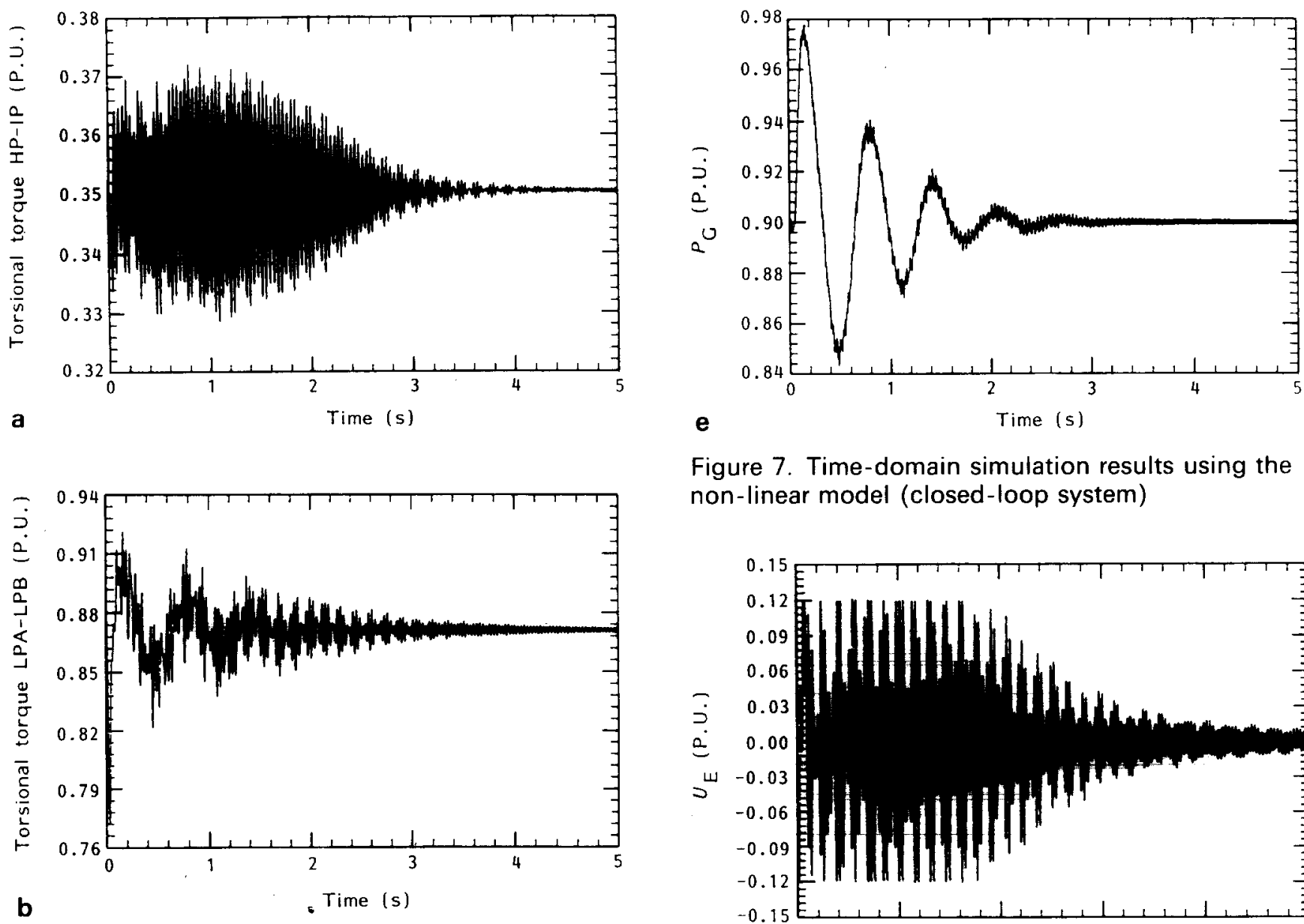

Figure 7. Time-domain simulation results using the non-linear model (closed-loop system)
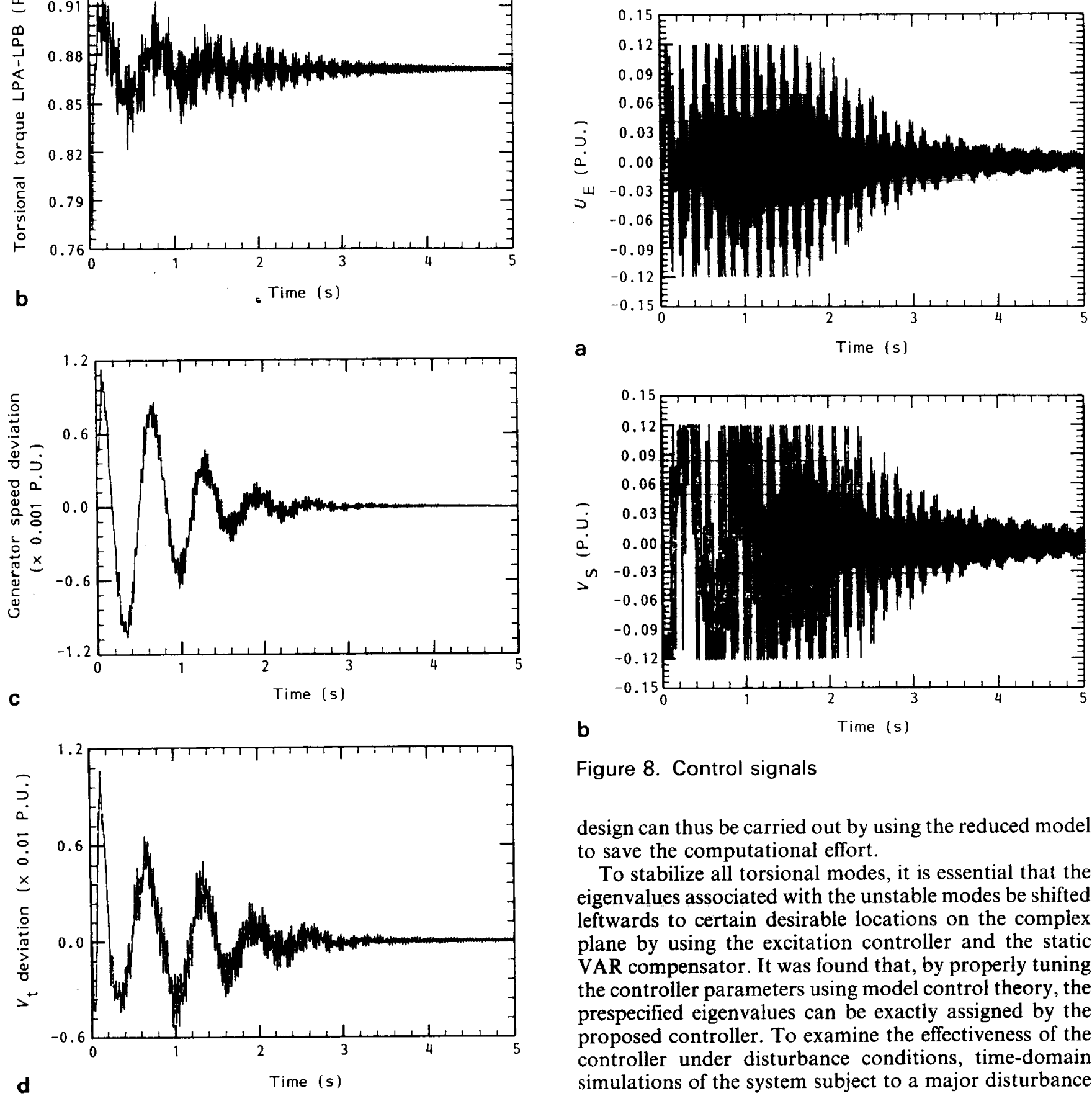

Figure 8. Control signals

design can thus be carried out by using the reduced model to save the computational effort.

To stabilize all torsional modes, it is essential that the eigenvalues associated with the unstable modes be shifted leftwards to certain desirable locations on the complex plane by using the excitation controller and the static VAR compensator. It was found that, by properly tuning the controller parameters using model control theory, the prespecified eigenvalues can be exactly assigned by the proposed controller. To examine the effectiveness of the controller under disturbance conditions, time-domain simulations of the system subject to a major disturbance 
were performed using a non-linear system model. It was found that all torsional modes can be stabilized by the developed controller.

Since the approach is generally applicable to any large-scale system, it can be easily extended to multi-machine power system problems.

\section{References}

1 IEEE SSR Working Group 'Countermeasures to subsynchronous resonance problems' IEEE Trans. Power Appar. \& Syst. Vol PAS-99 (1980) pp 1810-1818

2 Yan, A, Wvong, $M D$ and $Y u, Y N$ 'Excitation control of torsional oscillations' IEEE PES Summer Meeting, Vancouver, British Columbia (July 1979) Paper A79 505-9

3 Yan, $\mathbf{A}$ and $\mathrm{Yu}, \mathbf{Y} \mathbf{N}$ 'Multi-mode stabilization of torsional oscillation using output feedback excitation control' IEEE Trans. Power Appar. \& Syst. Vol PAS-101 (1982) pp 1245-1253

4 Balda, J C. Harley, R G and Eitelberg, E 'Damping of torsional shaft oscillations in two interacting neighboring turbogenerators' IEEE Trans. Energy Conversion Vol EC-2 No 1 (1987) pp 108-115

5 Ramey, D G, Dorney, J W, Kimmel, D S and Kroening, F H 'Dynamic stabilizer verification tests at the San Juan station' IEEE Trans. Power Appar. \& Syst. Vol PAS-100 (1981) pp 5011-5019

6 Kimmel, D S, Jones, W H, Carter, M P and Bednarek, J H 'Dynamic stabilizer on-line experience' IEEE Trans. Power Appar. \& Syst. Vol PAS-103 (1984) pp 72-75

7 Hammad, A E and El-Sadek, $M$ 'Application of a thyristor controlled VAR compensator for damping subsynchronous oscillations in power systems' IEEE Trans. Power Appar. \& Syst. Vol PAS-103 (1984) pp 198-212

8 Wang, $L$ and $\mathrm{Hsu}, \mathrm{Y} Y$ 'Damping of subsynchronous resonance using excitation controllers and static VAR compensators: a comparative study' IEEE PES Winter Meeting. New Orleans (1987) Paper 87WM 244-7

9 Hsu, Y Y and Wu, C J 'Design of PID static VAR controllers for the damping of subsynchronous oscillations' IEEE PES Summer Meeting, San Francisco (1987) Paper 87SM 417-9

10 Hsu, $Y Y$ and Wang, L'Damping of a parallel AC-DC power system using PID power system stabilizers and rectifier current regulators' IEEE PES Joint Power Generation Conference, Miami (1987) Paper 87JPGC 653-9

11 Hall, M C and Hodges, D A 'Experience with $500 \mathrm{kV}$ subsynchronous resonance and resulting turbine generator shaft damage at Mohave generating station' IEEE Publication 76CH 1066-O-PWR (1976) pp 22-29

12 IEEE Committee Report 'First Benchmark Model for computer simulation of subsynchronous resonance' IEEE Trans. Power Appar. \& Syst. Vol PAS-96 (1977) pp 1565-1572

13 Kokotovic, P V, Allemong, J J, Winkelman, J R and Chow, $\mathbf{J ~ H}$ 'Singular perturbation and iterative separation of time scales' Automatica Vol 16 (1980) pp 23-33

14 Winkelman, J R, Chow, J H, Allemong, J J and Kokotovic, P V 'Multi-time-scale analysis of a power system' Automatica Vol 16 (1980) pp 35-43

15 Brucoli, $\mathbf{M}$, Torelli, $\mathbf{F}$ and Trovato, $M$ 'A decentralized control strategy for dynamic shunt VAR compensation in interconnected power systems' Proc. IEE Vol 132 Part C (1985) pp 229-236

$16 \mathrm{Yu}, \mathrm{Y} \mathbf{N}$ Electric Power System Dynamics Academic Press, New York (1983)

17 Chen, $C \mathrm{~L}$ and Hsu, $Y \mathrm{Y}$ 'Coordinated synthesis of multimachine power system stabilizer using an efficient decentralized modal control algorithm' IEEE Trans. Power Syst. Vol PWRS-2 No 3 (1987) pp 543-551

18 Porter, B and Crossley, R Modal Control Jheory and Applications Taylor \& Francis (1972)

19 Liou, $\mathrm{K}$ L and Hsu, $Y \mathbf{Y}$ 'Damping of generator oscillations using static VAR compensators' IEEE Trans. Aerospace \& Electron. Syst. Vol AES-22 No 5 (1986) pp 605-617
Appendix 1. System parameters and operating conditions

The following parameters are all on an 892.4 MVA base $^{12}$.

Rotor mass-spring parameters ${ }^{2,3}$

\begin{tabular}{|c|c|c|c|c|}
\hline Mass & Shaft & $H(\mathrm{sec})$ & $\begin{array}{l}K \\
\text { (p.u. torque/rad) }\end{array}$ & $F(\%)$ \\
\hline $\mathrm{P}$ & & 0.092897 & & 30.0 \\
\hline & HP-IP & 0.155589 & 19.303 & 26.0 \\
\hline & IP-LPA & & 34.929 & \\
\hline $\mathrm{PA}$ & LPA-LPB & 0.858670 & 52.038 & 22.0 \\
\hline & LPB-GEN & 0.884215 & 70.858 & 22.0 \\
\hline$E N$ & GEN-EXC & $\begin{array}{l}0.868495 \\
0.0342165\end{array}$ & 2.822 & $\begin{array}{l}0.0 \\
0.0\end{array}$ \\
\hline
\end{tabular}

Speed governor system ${ }^{16}$

$K_{\mathrm{G}}=25.0 \quad T_{\mathrm{CH}}=0.3 \mathrm{~s}$

$T_{\mathrm{SR}}=0.2 \mathrm{~s} \quad T_{\mathrm{RH}}=7.0 \mathrm{~s}$

$T_{\mathrm{SM}}=0.3 \mathrm{~s} \quad T_{\mathrm{CO}}=0.2 \mathrm{~s}$

Exciter and voltage regulator ${ }^{2.3}$

$$
\begin{array}{lll}
K_{\mathrm{A}}=50.0 & T_{\mathrm{A}}=0.01 \mathrm{~s} & T_{\mathrm{E}}=0.002 \mathrm{~s} \\
E_{\mathrm{FDmax}}=7.3 & U_{\text {Emax }}=0.12 & \\
E_{\mathrm{FD} \min }=-7.3 & U_{\text {Emin }}=-0.12 &
\end{array}
$$

The synchronous generator (p.u.)

$$
\begin{array}{lccc}
R_{\mathrm{A}}=0.0015 & R_{\mathrm{F}}=0.001 & R_{\mathrm{kd}}=0.0037 \\
& \multicolumn{2}{c}{R_{\mathrm{fq}}=0.0053} & R_{\mathrm{kq}}=0.0182 \\
X_{\mathrm{d}}=1.79 & X_{\mathrm{ad}}=1.66 & X_{\mathrm{F}}=0.04 & X_{\mathrm{kd}}=0.006 \\
X_{\mathrm{q}}=1.71 & X_{\mathrm{aq}}=1.58 & X_{\mathrm{fq}}=0.115 & X_{\mathrm{kq}}=0.245
\end{array}
$$

Transformer and series-compensated transmission line

$R_{\mathrm{T}}=0.01$ p.u. $\quad X_{\mathrm{T}}=0.14$ p.u. $\quad R_{\mathrm{L}}=0.02$ p.u. $X_{\mathrm{L} 1}=0.50$. p.u. $\quad X_{\mathrm{L} 2}=0.06$ p.u. $\quad X_{\mathrm{C}} / X_{\mathrm{L}}=50 \%$

Static VAR compensator ${ }^{15,19}$

$$
\begin{array}{lll}
K_{\mathrm{S}}=1.0 & T_{\mathrm{S}}=0.15 \mathrm{~s} & X_{\mathrm{TS}}=0.08 \text { p.u. } \\
B_{\mathrm{C}}=0.6 \text { p.u. } & B_{\mathrm{L}}=B_{\mathrm{L} 0}+\Delta B_{\mathrm{L}} \quad B_{\mathrm{L} 0}=-0.45 \text { p.u. } \\
B_{\mathrm{Lmax}}=-0.42 \text { p.u. } & V_{\mathrm{S} \max }=0.12 \text { p.u. } \\
B_{\mathrm{Lmin}}=-0.48 \text { p.u. } & V_{\mathrm{S} \min }=-0.12 \text { p.u. }
\end{array}
$$

Operating conditions

$$
P_{\mathrm{G}}=0.9 \text { p.u. } \quad \mathrm{PF}=0.9 \text { lagging } \quad V_{\mathrm{t}}=1.05 \text { p.u. }
$$

\section{Appendix 2. Iterative equations for time-scale decomposition}

The time-scale decomposition method ${ }^{13,14}$ can be used to decouple the system

$$
\begin{aligned}
& \dot{\mathbf{X}}(t)=\mathbf{A X}(t)+\mathbf{B U}(t) \\
& \mathbf{Y}(t)=\mathbf{C X}(t)
\end{aligned}
$$

into two subsystems: 
- slow subsystem

$$
\dot{\mathbf{X}}_{1}(t)=\overline{\mathbf{A}}_{11} \mathbf{X}_{1}(t)+\overline{\mathbf{B}}_{1} \mathbf{U}(t)
$$

- fast subsystem

$$
\begin{aligned}
& \dot{\mathbf{X}}_{2}(t)=\overline{\mathbf{A}}_{22} \mathbf{X}_{2}(t)+\overline{\mathbf{B}}_{2} \mathbf{U}(t) \\
& \mathbf{Y}(t)=\overline{\mathbf{C}}_{2} \mathbf{X}_{2}(t)
\end{aligned}
$$

The matrices $\overline{\mathbf{A}}_{11}, \overline{\mathbf{B}}_{1}, \overline{\mathbf{A}}_{22}, \overline{\mathbf{B}}_{2}$ and $\overline{\mathbf{C}}_{2}$ can be solved iteratively using the following equations for the $j$ th iteration $^{13,14}$ :

$$
\begin{aligned}
& \overline{\mathbf{A}}_{11, k j}=\overline{\mathbf{A}}_{11, k}-H_{k j} \overline{\mathbf{A}}_{21, k} \\
& \overline{\mathbf{A}}_{12, k j}=\overline{\mathbf{A}}_{12, k}-H_{k j} \overline{\mathbf{A}}_{22, k}+\overline{\mathbf{A}}_{11, k j} H_{k j} \\
& \overline{\mathbf{A}}_{21, k j}=\overline{\mathbf{A}}_{21, k} \\
& \overline{\mathbf{A}}_{22, k j}=\overline{\mathbf{A}}_{22, k}+\overline{\mathbf{A}}_{21, k} H_{k j} \\
& \overline{\mathbf{B}}_{1, k j}=\overline{\mathbf{B}}_{1, k}-H_{k j} \overline{\mathbf{B}}_{2, k} \\
& \overline{\mathbf{B}}_{2, k j}=\overline{\mathbf{B}}_{2, k} \\
& \overline{\mathbf{C}}_{2, k j}=\overline{\mathbf{C}}_{2, k}+\overline{\mathbf{C}}_{1, k} H_{k j}
\end{aligned}
$$

$H_{k j}$ can be computed iteratively by solving the equations

$$
\begin{aligned}
& H_{k i}=\mathbf{A}_{12} \overline{\mathbf{A}}_{22, k}^{-1}+\left(\overline{\mathbf{A}}_{11, k}-H_{k, i-1} \overline{\mathbf{A}}_{21, k}\right) H_{k, i-1} \overline{\mathbf{A}}_{22, k}^{-1} \\
& H_{k 1}=\overline{\mathbf{A}}_{12, k} \overline{\mathbf{A}}_{22, k}^{-1}
\end{aligned}
$$

and the matrices in the $k$ th iteration, $\overline{\mathbf{A}}_{11, k}, \overline{\mathbf{A}}_{12, k}, \overline{\mathbf{A}}_{21, k}$, $\overline{\mathbf{A}}_{22, k}, \overline{\mathbf{B}}_{1, k}, \overline{\mathbf{B}}_{2, k}$ and $\overline{\mathbf{C}}_{2, k}$, can be obtained from the equations

$$
\begin{aligned}
& \overline{\mathbf{A}}_{11, k}=\mathbf{A}_{11}-\mathbf{A}_{12} L_{k} \\
& \overline{\mathbf{A}}_{12, k}=\mathbf{A}_{12} \\
& \overline{\mathbf{A}}_{21, k}=\mathbf{A}_{21}-\mathbf{A}_{22} L_{k}+L_{k} \overline{\mathbf{A}}_{11, k} \\
& \overline{\mathbf{A}}_{22, k}=\mathbf{A}_{22}+L_{k} \mathbf{A}_{12} \\
& \overline{\mathbf{B}}_{1, k}=\mathbf{B}_{1} \\
& \overline{\mathbf{B}}_{2, k}=\mathbf{B}_{2}+L_{k} \mathbf{B}_{1} \\
& \overline{\mathbf{C}}_{2, k}=\mathbf{C}_{2}
\end{aligned}
$$

where $\mathbf{A}_{11}, \mathbf{A}_{12}, \mathbf{A}_{21}$ and $\mathbf{A}_{22}$ are submatrices of the matrix $\mathbf{A}$ and $L_{k}$ is computed iteratively by solving the equation

$$
\begin{aligned}
& L_{i}=\mathbf{A}_{22}^{-1} \mathbf{A}_{21}+\mathbf{A}_{22}^{-1} L_{i-1}\left(\mathbf{A}_{11}-\mathbf{A}_{12} L_{i-1}\right) \\
& L_{1}=\mathbf{A}_{22}^{-1} \mathbf{A}_{21}
\end{aligned}
$$

